Research Paper

\title{
Selective amplification of hypermethylated DNA from diverse tumor types via MSRE-PCR
}

\author{
Karen B. Chapman ${ }^{1}$ and Brandon W. Higgs ${ }^{1,2}$ \\ ${ }^{1}$ Center for Biotechnology Education, Krieger School of Arts and Sciences, Johns Hopkins University, Baltimore, MD 21218, \\ USA \\ ${ }^{2}$ Genmab, Princeton, NJ 08540, USA
}

Correspondence to: Karen B. Chapman, email: kchapma3@jhu.edu

Keywords: MSRE-PCR; CDMR; DNA methylation biomarkers; epigenetics; cancer

Received: August 21, $2020 \quad$ Accepted: November 12, $2020 \quad$ Published: November 24, 2020

Copyright: @ 2020 Chapman and Higgs. This is an open access article distributed under the terms of the Creative Commons Attribution License (CC BY 3.0), which permits unrestricted use, distribution, and reproduction in any medium, provided the original author and source are credited.

\section{ABSTRACT}

DNA methylation biomarkers are increasingly utilized for the detection, prognosis and monitoring of cancer. Here we use publicly-available whole genome bisulfite sequencing data to identify differentially methylated regions (cDMRs) in diverse tumor types and further define a set of genomic target regions that have optimal characteristics for Methylation Sensitive Restriction Enzyme-PCR (MSREPCR)-based detection: conserved hypermethylation in tumors, abundant MSRE sites and low methylation levels in normal tissues. The identified MSRE-PCR target regions $(n=1,294)$ were primarily encompassed within CpG islands $(97 \%)$ and promoters $(81 \%)$ with $39 \%$ of the target regions overlapping the transcription start site. Gene set enrichment analysis of the target regions identified significant enrichment of genes involved in neuronal development. A multiplexed MSRE-PCR assay was developed interrogating 47 target regions and was tested on a set of genomic DNAs $(n=100)$ from diverse tumor and normal tissue types including colon, breast, lung, stomach and blood. A logistic regression model containing seven target region amplicons distinguished between tumor and normal tissue in the training $(n=50)$ with a ROC AUC of $0.97(95 \%$ CI $[0.92,1])$ and independent test set $(n=50)$ with an AUC of $0.93(95 \% \mathrm{CI}[0.84,1])$. These findings show that genomic regions with conserved hypermethylation across diverse tumor types, abundant MSRE sites and low methylation levels in normal tissues provide target regions for the detection of tumor DNA via MSRE-PCR. The selective amplification of tumor-derived DNA via MSRE-PCR may have utility in the development of non-invasive cancer detection and surveillance strategies.

\section{INTRODUCTION}

Global epigenetic changes, including DNA methylation, are widely regarded as a hallmark of cancer [1]. Alterations include a decrease in overall CpG methylation levels coupled with discrete regions of hypermethylation, typically localized in the promoterassociated $\mathrm{CpG}$ islands. Hypermethylation has been associated with cancer progression [2] and the silencing of growth regulating genes and tumor suppressor genes [3]. As a result, a growing number of DNA methylation biomarkers are being utilized in the development of novel assays for monitoring cancer progression $[4,5]$, treatment response [6] and early detection [7].

Multiple methods to detect DNA methylation of $\mathrm{CpG}$ dinucleotides have been described. Bisulfite treatment of DNA converts unmethylated cytosines to uracil, a property that can be used to detect genomewide $\mathrm{CpG}$ methylation at single nucleotide resolution via Whole Genome Bisulfite Sequencing (WGBS) [8]. PCR methodologies such as Methylation Specific PCR (MSP) also employ bisulfite conversion as well as two sets of PCR primers that distinguish between methylated and unmethylated version of a selected amplicon $[4,9]$. 
Alternatively, without employing bisulfite conversion, selective amplification of differentially methylated regions can be achieved by Methylation Sensitive Restriction Enzyme PCR (MSRE-PCR) [10]. PCR primer-binding sites are designed to flank genomic regions that have a differentially methylated $\mathrm{CpG}$ encompassed within a methylation-sensitive restriction site. Methylated DNA is protected from MSRE digestion, thereby allowing selectively amplification. Additionally, MSRE-PCR can be multiplexed to interrogate multiple target regions simultaneously [11] and applications of this technology are being explored for colorectal cancer detection [12] and lung cancer detection [13]. For clinical applications, MSRE-PCR is a desirable strategy in that it avoids bisulfite conversion, a chemical treatment that can result in DNA damage and loss [11]. Preserving the integrity and complexity of the DNA in clinical samples is particularly relevant in the analysis of cfDNA, where sample quantities are low and the fraction of tumorderived DNA can be limiting.

The selective amplification and detection of tumorderived DNA via MSRE-PCR requires the targeted amplicon to have MSRE site(s) and to be hypermethylated. In theory, the most optimal amplicons for a highly specific MSRE-PCR assay would be fully unmethylated in normal DNA, and thus a complete digestion would preclude amplification of the normal tissue-derived DNA, facilitating the selective amplification of tumor-derived DNA.

Here we describe a bioinformatics strategy identifying genomic regions that incorporate these criteria to optimize MSRE-PCR assays. We identify amplicons with extremely low methylation levels $<2.5 \%$ in normal tissues, conserved hypermethylation in diverse tumors and abundant MSRE sites. Furthermore, we demonstrate the feasibility of using these identified target regions in a multiplexed MSRE-PCR assay in an independent cohort of genomic DNAs from diverse tumors and normal tissues derived from lung, breast, stomach, colon and blood. This strategy may provide a path for sensitive and specific assays for non-invasive cancer diagnostics.

\section{RESULTS}

\section{Identification of cDMRs}

To identify cancer-associated Differentially Methylated Regions (cDMRs), publicly available WGBS datasets from a diverse cohort of normal tissues and tumors were analyzed (Table 1). The normal samples used in this analysis were comprised of 5 different tissue types (liver, lung, breast, colon and blood-derived B-cells) in an effort to simulate the epigenetic diversity in methylation patterns that would be seen in normal DNA. The cancer samples were equally diverse representing lung, liver and colon primary tumors as well as cell lines derived from prostate and breast tumors. In total, 18 WGBS samples representing normal tissues $(n=9)$ and diverse primary tumors and tumor cell lines $(n=9)$ were obtained from the NCBI Sequence Read Archive (SRA) database. In an effort to maximize sequencing coverage and interrogate as much of the genome as possible, multiple runs for individual biological samples were combined to yield an average coverage that exceeded 11-fold. The 48 SRA runs encompassed in this analysis are detailed in Supplementary Table 1.

\section{Global CpG methylation characteristics}

The global genomic $\mathrm{CpG}$ methylation levels and patterns in this cohort were characterized to understand the context of differential methylation. Comparing global $\mathrm{CpG}$ methylation levels between the cancer and normal samples, the percent methylation in the cancer samples was significantly lower and more variable than the normal sample group $(p=0.005)$. The normal samples had a mean $\mathrm{CpG}$ methylation of $67.4 \%$, compared to $54.5 \%$ in the cancer samples (Figure 1A). Consistent with the variability observed in the global $\mathrm{CpG}$ methylation levels in the cancer samples, principal component analysis (PCA) and Pearson's correlation analysis also indicated that the cancer samples were more diverse, with the normal samples having an average Pearson's $\mathrm{r}$ $=0.77$ versus 0.67 in the cancers (Figure $1 \mathrm{~B}$ and $1 \mathrm{C}$ ). Despite the diversity of tissue and tumor types in this WGBS discovery cohort, clustering based on global CpG methylation grouped the majority of the cancer samples together (Figure 1D).

\section{Identification of cDMRs}

Identification of cDMRs was conducted with Metilene [14] using parameters optimized for the identification of regions that would be in a size range suitable for PCR and have multiple differentially methylated CpGs. This analysis yielded 195,590 cDMRs, the majority of which were hypomethylated in cancer (95\%), consistent with the lower overall level of $\mathrm{CpG}$ methylation observed in the cancer sample group relative to normal (Figure 2A). The genomic location of both the hypermethylated and hypomethylated cDMRs appeared relatively evenly distributed across the chromosomes (Figure 2B). The average size of the identified cDMRs was $885 \mathrm{nt}$ with a mean of $33 \mathrm{CpGs}$, although some regions exceeded $5 \mathrm{~kb}$ with over $200 \mathrm{CpGs}$ (Figure 2C). The more prevalent hypomethylated cDMRs also had a greater maximum methylation difference, with some cDMRs exceeding a mean methylation difference of $60 \%$, compared to a maximum of $48 \%$ for the less abundant hypermethylated cDMRs (Figure 2D). 
Table 1: WGBS samples used in this study

\begin{tabular}{|c|c|c|c|c|c|c|}
\hline Sample Type & Name & $\begin{array}{l}\text { SRA Sample } \\
\text { and Reference }\end{array}$ & Description & SRA Runs & Mbytes & Reference \\
\hline \multirow{9}{*}{$\begin{array}{l}\text { Normal } \\
\text { Tissues }\end{array}$} & N3 & SRS1351498 & normal liver & 2 & 22,600 & {$[36]$} \\
\hline & N4 & SRS1352201 & normal liver & 2 & 12,108 & [36] \\
\hline & N5 & SRS1352208 & normal lung & 1 & 20,202 & [36] \\
\hline & N6 & SRS1353345 & normal lung & 1 & 26,014 & {$[36]$} \\
\hline & N7 & SRS1353348 & normal lung & 1 & 12,576 & {$[36]$} \\
\hline & N8 & SRS1352204 & normal liver & 1 & 24,195 & {$[36]$} \\
\hline & N9 & SRS505928 & normal colon & 5 & 27,840 & [37] \\
\hline & N10 & SRS505949 & normal B-cells & 3 & 36,975 & [37] \\
\hline & N11 & SRS505934 & normal breast & 5 & 29,531 & {$[37]$} \\
\hline \multirow{9}{*}{$\begin{array}{l}\text { Tumors and } \\
\text { Tumor Cell } \\
\text { Lines }\end{array}$} & $\mathrm{C} 3$ & SRS1352199 & liver tumor & 2 & 16,983 & [36] \\
\hline & $\mathrm{C} 4$ & SRS1352202 & liver tumor & 2 & 13,510 & {$[36]$} \\
\hline & $\mathrm{C} 5$ & SRS1353344 & lung tumor & 1 & 20,783 & [36] \\
\hline & C6 & SRS1353347 & lung tumor & 2 & 24,795 & [36] \\
\hline & $\mathrm{C} 7$ & SRS1353349 & lung tumor & 1 & 12,894 & [36] \\
\hline & $\mathrm{C} 8$ & SRS1352206 & liver tumor & 2 & 24,173 & {$[36]$} \\
\hline & C9 & SRS505929 & colon tumor & 6 & 36,391 & [37] \\
\hline & $\mathrm{C} 10$ & SRS505931 & prostate cancer cell line & 6 & 38,854 & [37] \\
\hline & $\mathrm{C} 11$ & SRS505933 & breast cancer cell line & 5 & 42,117 & [37] \\
\hline
\end{tabular}

\section{Identification of target regions for MSRE-PCR}

The optimal target region for selective amplification of tumor-derived DNA via MSRE-PCR is a a region methylated in cancer and unmethylated in normal, as this allows for the selective digestion of normal DNA with restriction enzymes that are inhibited by $\mathrm{CpG}$ methylation in the recognition site. With these considerations in mind, the 10,019 hypermethylated cDMRs were filtered for those with a mean methylation less than $2.5 \%$ in the normal samples, yielding 1,294 target hypermethylated cDMRs (target regions) (Figure 3A and 3B). As a group, the target regions had an average methylation level of $1.5 \%$ in the normal samples and $18.6 \%$ in the cancers (Figure 3C). The target regions, with an average length of $308 \mathrm{nt}$ and $34 \mathrm{CpGs}$ were smaller and more CpG-dense than the unfiltered cDMRs.

\section{Target regions had multiple methylation- sensitive restriction sites}

The selective amplification of target regions via MSRE-PCR requires the presence of MSRE recognition sites that allow for the selective digestion of unmethylated DNA. Here we focused on 5 MSREs: HhaI, HpaII, HpyCH4IV, AciI and BstUI, as each of these enzymes have a 4 base pair (bp) recognition sequence containing $\mathrm{CpG}$ and thus sites for these MSREs should be relatively abundant in CpG-rich regions. Indeed, all of the target regions had one or more of these 5 MSRE recognition sites (Figure 4A). The target regions had on average 19 MSRE sites and $34 \mathrm{CpGs}$ and thus more than half of the individual CpGs in these target regions were within recognition sites of one of the five MSREs. The relationship between the number of $\mathrm{CpGs}$ in the target regions and the number of MSREs was linear $\left(\mathrm{R}^{2}=0.89, p<0.0001\right)$ (Figure 4B).

\section{Target regions were primarily in promoters and CpG islands}

Target regions were not selected based on genomic context or proximity to known genes, rather on optimal amplicon characteristics that would allow for the most selective amplification via MSRE-PCR. These features included size, number of CpGs, low background methylation in normal tissues, and significant methylation across a diverse spectrum of tumor samples. Since the methodology was unbiased with respect to genomic features, we chose to look at the genomic context of the DMRs and selected target regions with respect to known genes, transcripts, $\mathrm{CpG}$ islands and shores flanking the $\mathrm{CpG}$ islands. The hypomethylated DMRs were located primarily in intergenic regions with only $5 \%$ in gene promoters, whereas the hypermethylated DMRs occurred primarily in promoters (Figure 5A). The target regions, which were a subset of the hypermethylated DMRs, showed an even more pronounced enrichment for promoter regions with $81 \%$ of the 1,294 target regions 
located in the promoter. The mean distance to the promoter was $57 \mathrm{nt}$, with $39 \%$ of the target regions overlapping the Transcription Start Site (TSS) (Supplementary Figure 1).

Target regions were located almost exclusively in CpG islands (97\%) whereas a lower fraction (84\%) of the hypermethylated DMRs were in $\mathrm{CpG}$ islands (Figure 5B). This contrasted with the hypomethylated DMRs, 94\% of which were located in other regions outside of $\mathrm{CpG}$ islands and shores.

\section{Target regions were enriched for neuronal genes}

Promoter methylation in cancer is largely regarded as being associated with repressive chromatin marks and gene silencing [3]. Since the vast majority of target regions were located in the promoter and $39 \%$ overlapped the TSS of known genes, we looked at the function and cellular location of the gene products encoded by the genes closest to the target regions to gain insight into the biological context of the cancer-associated differential methylation observed in these regions. Some of the target regions

A

Percent Methylation of CpGs

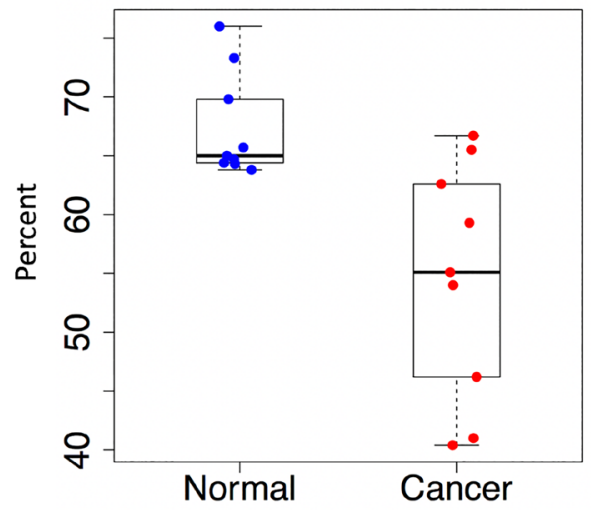

C Correlation Plot: Global CpG Methylation

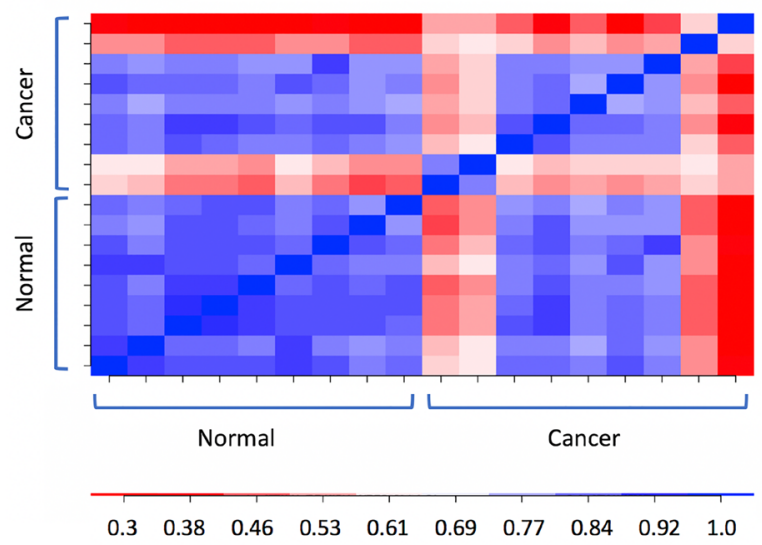

were adjacent to each other and/or closest to the same gene. Consequently, annotating the 1,293 target regions for the closest genes and then removing redundancies resulted in a reduced list of 562 genes, 39\% of which overlapped with the TSS. Functional enrichment analysis using Gene Ontology (GO) molecular function showed significant enrichment for DNA-binding transcription factor activity (FDR-corrected $p$-value 4.3e-11). Analysis of GO:biological processes and GO:cellular components showed concordant enrichment of neuronal function, with significant biological process terms being dominated by neuronal differentiation (FDR-corrected $p$-value 3.9e14), generation of neurons (FDR-corrected $p$-value 6.1e-14), and the top cellular component terms being glutamatergic synapse (FDR-corrected $p$-value 1.6e-15) and synaptic membrane (FDR-corrected $p$-value 3.1e-13). Since there were a large number of significant GO terms identified, the list of terms was further analyzed in Revigo to summarize clusters of semantically similar GO terms [15]. This analysis also underscored the neuronal theme with the most significant cluster terms being neuronal
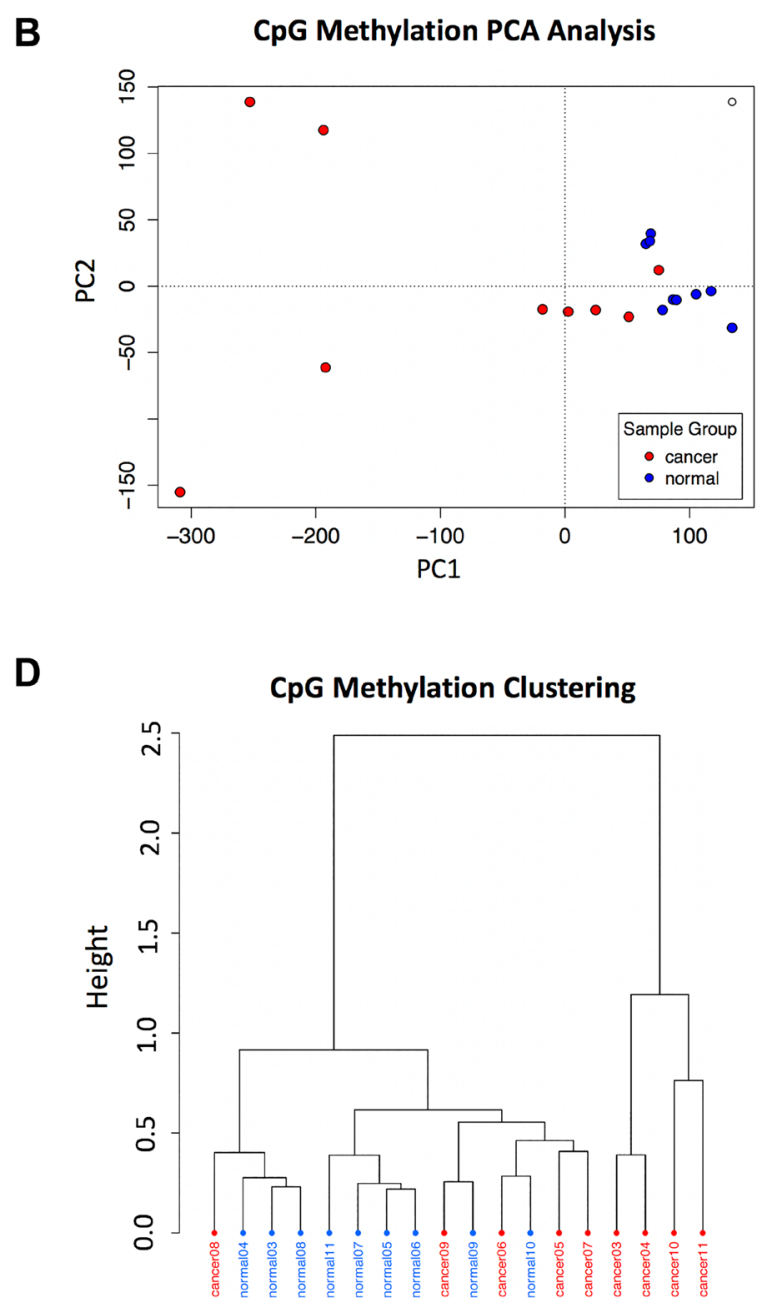

Figure 1: Global methylation characteristics in the WGBS dataset. (A) Percent methylation in the sample groups: normal $(n=$ 9) and cancer $(n=9)$. (B) CpG methylation PCA analysis. Cancer samples are shown in red and normal samples are in blue. (C) Pearson correlation plot of global $\mathrm{CpG}$ methylation. (D) $\mathrm{CpG}$ methylation clustering; distance method: correlation, clustering method: ward. 
differentiation for GO:biological process and synapse for GO:cellular component (Figure 6A and 6B).

\section{Hierarchical clustering}

Unsupervised hierarchical clustering of the mean methylation in the target regions across the samples shows that hypermethylation of many of these target regions were shared across multiple tumor samples and cancer types, yielding a clustering of the the cancer samples together, distinct from the normal samples which exhibit only low levels of methylation in these regions (Figure 7).

\section{Multiplexed MSRE-PCR}

To test the suitability of the target regions for MSRE-PCR based cancer detection, a multiplexed MSREPCR assay was developed and tested on genomic DNAs

\section{A Hyper and Hypomethylated cDMRs}

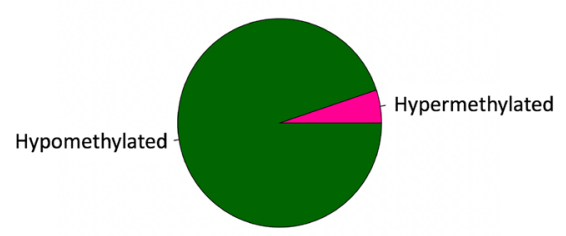

C

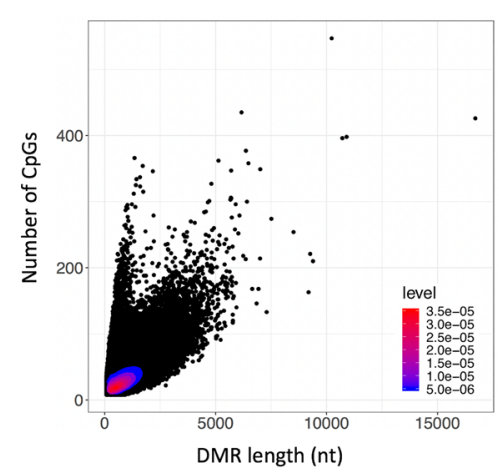

B
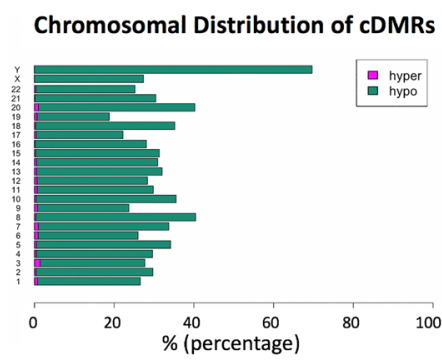

D

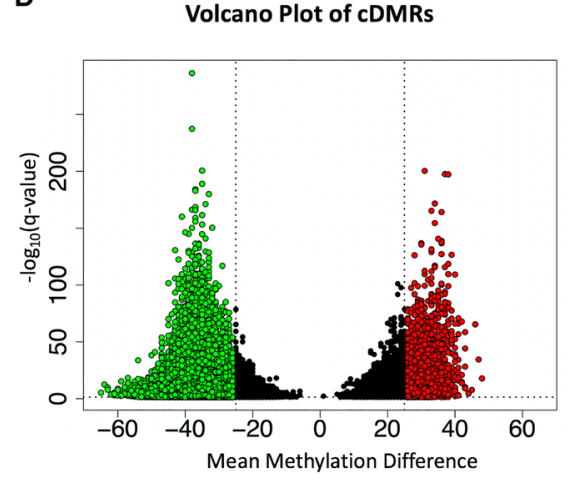

Figure 2: Cancer-associated Differentially Methylated Regions (cDMRs) (A) The majority (95\%) of the cDMRs are hypomethylated with only 5\% hypermethylated in cancer. (B) Chromosomal distribution of hypermethylated and hypomethylated cDMRs (C) Scatterplot of cDMR length versus number of CpGs, with the density of points indicated via heatmap. (D) Volcano plot of the cDMRs showing the mean methylation difference versus the - $\log _{10} q$-value. Hypermethylated cDMRs with a $q$-value $<0.05$ and mean methylation difference $>25 \%$ are indicated in red and hypomethylated cDMRs in green.

A

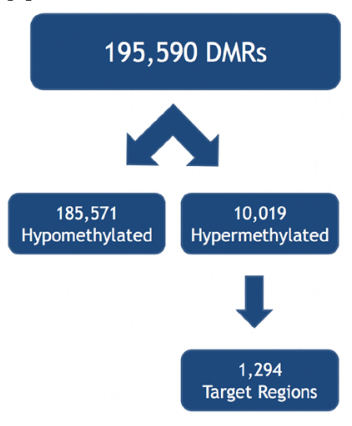

B

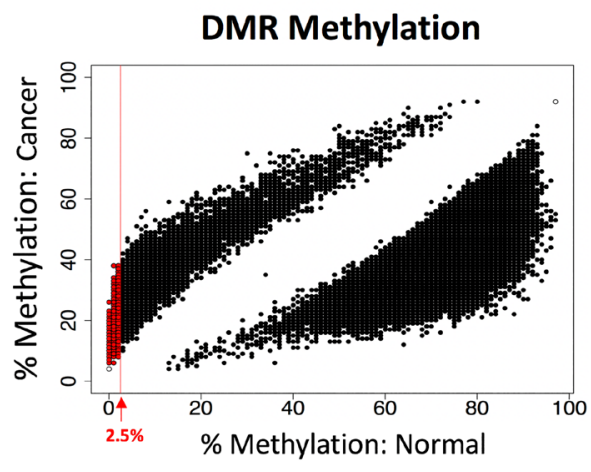

C

Target Region Methylation

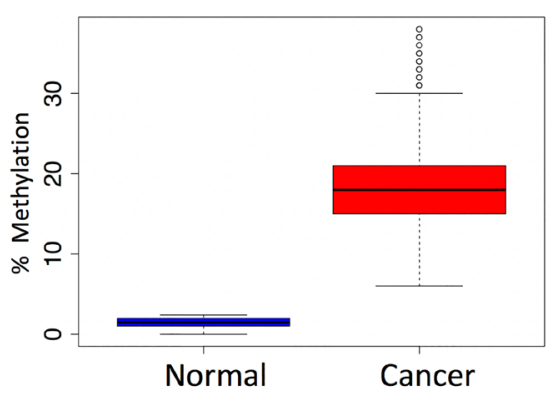

Figure 3: Selection of target regions. (A) The 195,590 cDMRs are separated into hypomethylated $(n=185,571)$ and hypermethylated $(n=10,019)$. The hypermethylated cDMRs are filtered for hypermethylated target regions with a mean methylation level $<2.5 \%$ in the normal samples, yielding 1,294 target regions. (B) A scatterplot of all 195,590 cDMRs showing the percent methylation in normal tissues versus the percent methylation in the cancer samples. The red line shows a mean methylation level $=2.5 \%$ in the normal samples. The 1,294 cDMRs passing the filtering criteria (target regions) are shown in red. (C) Average methylation levels in the target regions is $1.5 \%$ in the normal samples $(n=9)$ and $18.6 \%$ in the cancer samples $(n=9)$. 
derived from tumors and normal tissues. The target regions were sorted based on $q$-value and PCR primers for a panel of 47 target amplicons and 3 controls were designed for a multiplexed MSRE assay (Supplementary Table 2). The MSRE assay was carried out by first digesting the DNA samples with 5 MSREs followed by multiplexed end-point PCR with the panel of 50 primer pairs. Quantitation of the individual amplicons was carried out by sequencing the amplification products, quantitating mapped reads and normalizing to an internal control amplicon that was not differentially methylated in the WGBS discovery cohort and thus predicted to amplify consistently across sample types with diverse methylation patterns (Supplementary Table 2).

The performance of the multiplexed MSRE-PCR assay was assessed on an independent, diverse cohort of genomic DNA samples $(n=100)$ encompassing tumor and normal samples from lung, breast, stomach and colon as well as normal peripheral blood leukocytes (Table 2 and Supplementary Table 5). This independent cohort of DNAs is comprised of different samples than were in the WGBS discovery cohort and notably contains several tissue types that were not encompassed in discovery cohort including peripheral blood leukocytes, normal stomach tissue and stomach tumors. The dataset was randomly partitioned into training $(n=50)$ and testing $(n=50)$ sets for predictive modeling.

The performance of the multiplexed MSRE-PCR assay in the training set was assessed by comparing the relative levels of amplification in the two sample groups. Amplicons with fewer than 10 reads per sample on average were removed from the analysis $(n=12)$ leaving 35 amplicons. As shown in the volcano plot in Figure 8A, all 35 of the amplicons had $>2$-fold elevated amplification in the tumor DNA samples relative to the normal tissue DNA samples, with 27 exhibiting $>10$-fold. Most of the amplicons (21/35) of exhibited significant elevation in

\section{A MSRE Sites in Target Regions}

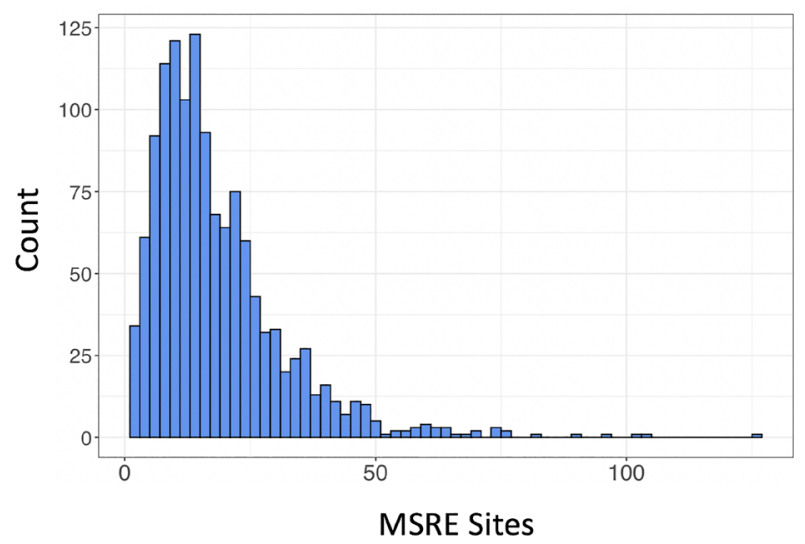

tumor samples (FDR corrected $p$-value $<0.05$, MannWhitney $U$ Test). Visualization of these 21 amplicons via hierarchical clustering shows that most of the tumor DNA samples have elevated amplification levels of multiple amplicons relative to the normal tissue DNAs (Figure 8B). Additionally, none of the amplicons are specific for a single tumor type; most are broadly elevated in a subset of samples across the four tumor types surveyed here: breast, lung, colon and stomach tumors. The Receiver Operating Characteristic (ROC) area under the curve (AUC) for individual amplicons in the training set ranged from 0.54 to 0.89 , with 9 amplicons having an $\mathrm{AUC}>0.8$ in the training set (Supplementary Table 6).

\section{MSRE-PCR panel modeling and prediction of cancer status}

The MSRE-PCR training set $(n=50)$ was used to develop a predictive model and also explore the minimum number of amplicons that are required to accurately classify sample DNAs as either tumor or normal tissue. The $\mathrm{R}$ package glmnet [16] was used to develop a logistic regression model using a lasso penalty for feature selection. This analysis yielded a 7-marker model that performed with a ROC AUC in the training set of 0.97 $(95 \%$ CI $[0.92,1])$. The performance of this 7-marker model was validated in the independent test set $(n=50)$, yielding an AUC of 0.93 (95\% CI $[0.84,1])$ (Figure 9). The 7 markers used in this model correspond to amplicons that are contained within the gene or upstream promoter regions of $A N K R D 33 B, C H S T 2, S P O N 1, P P P 2 R 5 C$, KCNG2, KIAA1522 and TH2LCRR.

Visualization of a representative target region in the $A N K R D 33 B$ gene is shown in Figure 10A. Three identified hypermethylated cDMRs are shown, with the WGBS tracks illustrating the promoter hypermethylation in the cancer samples and the relatively low levels of

\section{B CpGs vs. MSRE Sites}

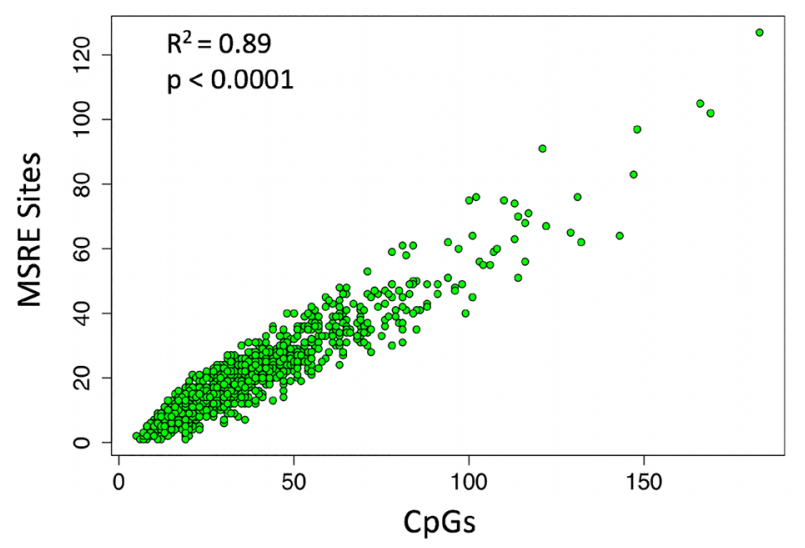

Figure 4: Methylation sensitive restriction sites in target cDMRs. Five MSREs are considered here: HhaI, HpaII, HpyCH4IV, AciI and BstUI. (A) Histogram of the number of MSRE sites in the target regions. (B) Scatterplot of the number of MSRE sites versus the number of CpGs in the target regions showing a linear relationship $\left(\mathrm{R}^{2}=0.89, p<0.0001\right)$. 
Table 2: Genomic DNA samples used in MSRE-PCR assay

\begin{tabular}{lcc}
\hline Tissue & Tumors & Normal \\
\hline Lung & 12 & 8 \\
Breast & 12 & 8 \\
Colon & 12 & 8 \\
Stomach & 12 & 8 \\
Peripheral Blood Leukocytes & 0 & 20 \\
\hline
\end{tabular}

methylation in the normal tissues in the discovery cohort. Figure 10B shows a close-up of the 106-bp MSRE-PCR amplicon within a target region in the $A N K R D 33 B$ gene containing $9 \mathrm{CpGs}, 7$ of which overlap with an MSRE site.

\section{DISCUSSION}

Here we describe the identification of cDMRs in diverse tumor types using publicly available WGBS data and further define a set of 1,294 target regions with optimal characteristics for MSRE-PCR-based detection of tumor DNA. The utility of these target regions in the context of MSRE-PCR is shown here with a multiplexed assay demonstrating that all 35 amplicons were $>2$-fold elevated in tumor-derived DNA relative to normal tissue DNA. In a diverse cohort of tumor and normal tissue DNAs, most of the individual MSRE-amplicons (21/35) showed significantly elevated amplification in tumorderived DNA versus normal (FDR-corrected $p<0.05$ ). Furthermore, despite the diversity of tumor and tissue types in the MSRE-PCR cohort, a logistic regression model using 7 of the amplicons distinguished between tumor versus normal tissue with an AUC of 0.97 in the training set and 0.93 in the independent test set. This result underscores the conservation of hypermethylation in these regions across diverse tumor types and also demonstrates the utility of using MSRE-PCR to detect tumor-derived DNA.

In the analysis of the WGBS discovery cohort, we observed that overall $\mathrm{CpG}$ methylation levels were lower and more variable in the tumors and this global loss of methylation was punctuated by local regions of hypermethylation, an observation consistent with multiple reports in the literature $[2,17]$. We further defined target regions for MSRE-PCR, a subset of the hypermethylated cDMRs that had low background methylation in normal tissues. Although the WGBS methodology used to identify targets was genome-wide with an average of 11-fold coverage and hence relatively unbiased with respect to genomic features, we observed significant enrichment
A

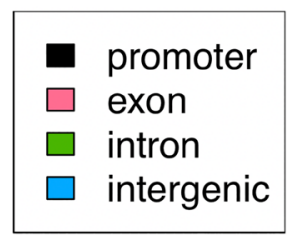

Hypomethylated cDMRs

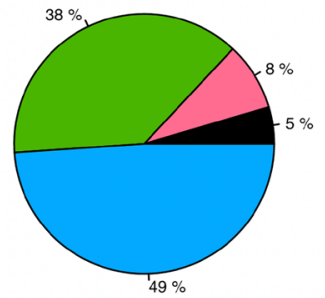

B

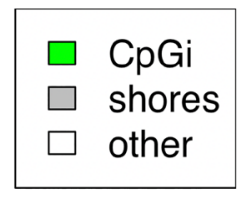

Hypomethylated cDMRs

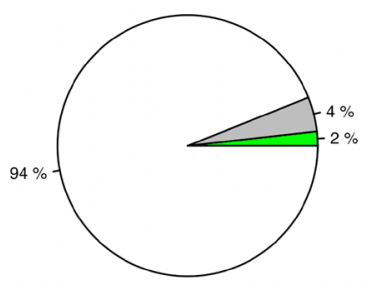

Hypermethylated cDMRs

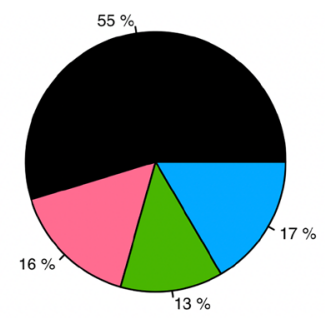

Hypermethylated cDMRs

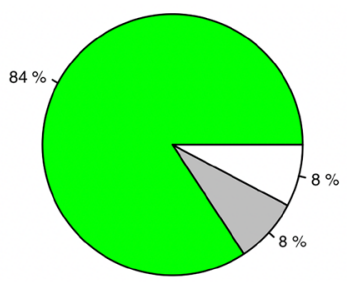

\section{Target Regions}

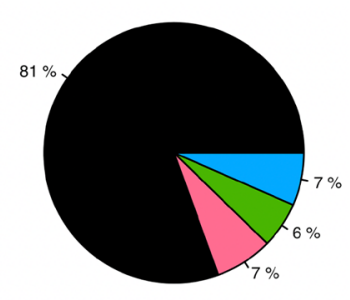

Target Regions

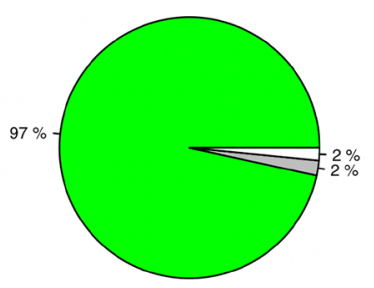

Figure 5: Target regions are enriched for promoter regions and CpG Islands. (A) hypomethylated cDMRs, hypermethylated cDMRs, and target regions, a subset of the hypermethylated cDMRs, are annotated with gene parts: promoter, exon, intron or intragenic. $81 \%$ of target regions are in promoters. (B) cDMRs are annotated with respect to CpG Islands (CpGi), shores or other. $97 \%$ of target regions are in a $\mathrm{CpGi}$. 
A

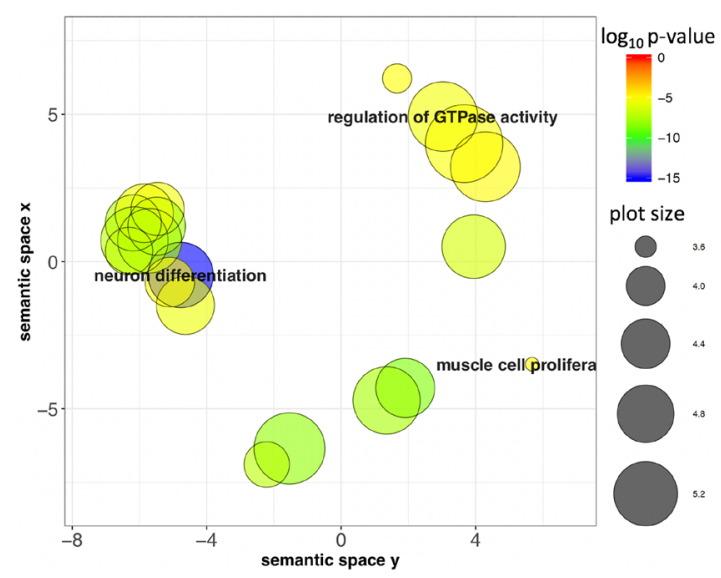

B

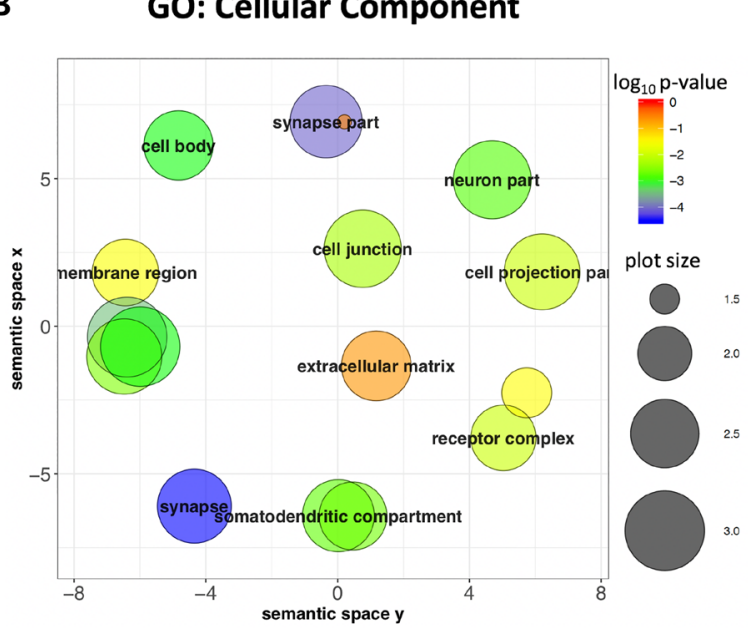

Figure 6: Genes closest to or overlapping target regions are enriched for Neuronal genes. (A) GO: Biological Process terms visualized in a semantic similarity-based scatterplot derived in Revigo indicates that neuron differentiation is the most significant term. (B) The most significant GO: Cellular Component terms are synapse and synapse part.

\section{Hierarchical Clustering: Target Region Methylation}

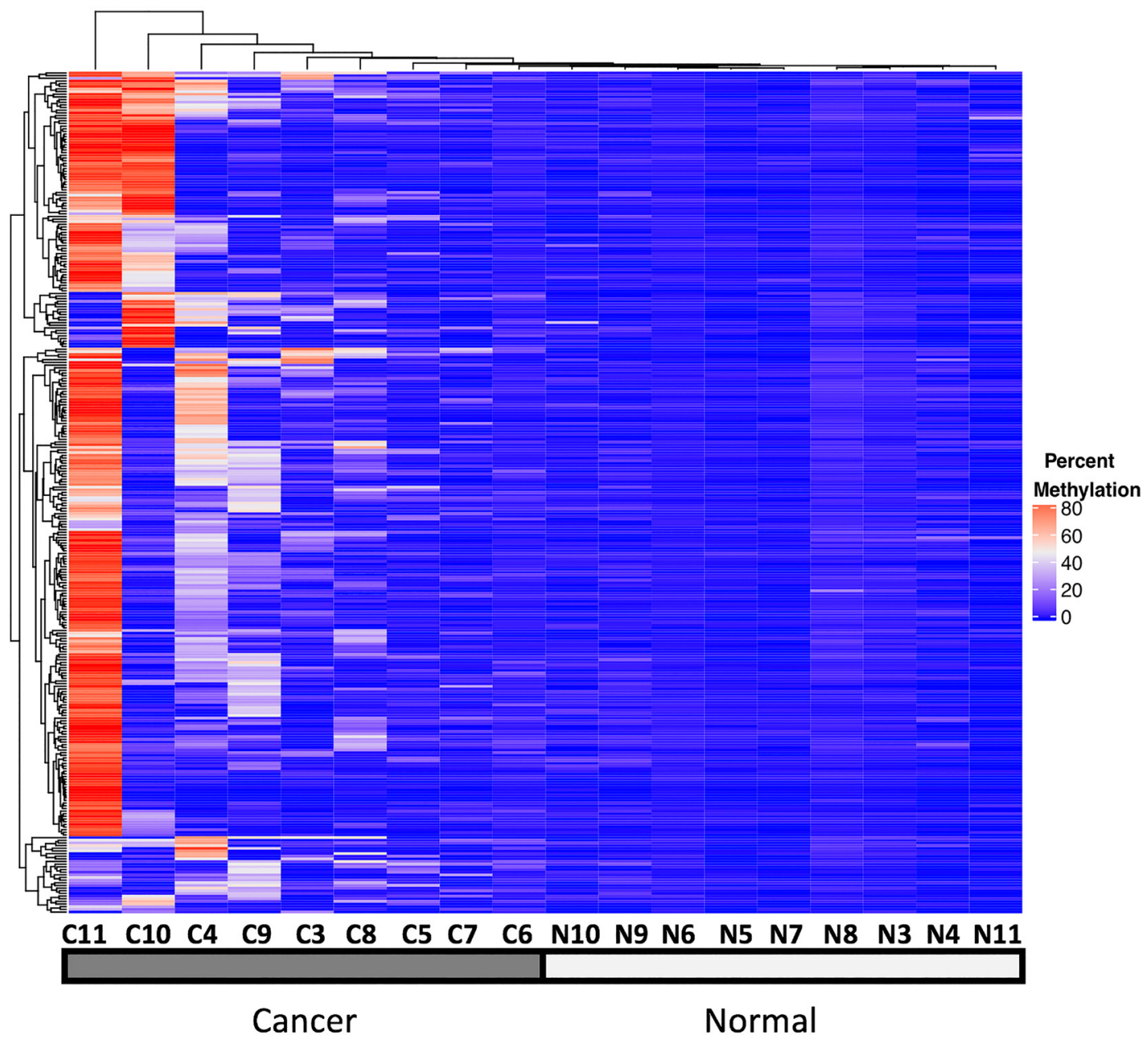

Figure 7: Unsupervised hierarchical clustering of target region methylation in all 18 WGBS samples used in this analysis. Percent methylation is indicated with the color gradient. 
of target regions in gene regulatory regions with $81 \%$ in promoters and $97 \%$ in $\mathrm{CpG}$ islands. Furthermore, these target regions were very proximal to, or overlapping the TSS with a mean distance of $57 \mathrm{nt}$ and $39 \%$ overlapping the TSS. This was in stark contrast to the hypomethylated DMRs with only $5 \%$ in promoters and only $2 \%$ in $\mathrm{CpG}$ islands, perhaps reflecting the global loss of $\mathrm{CpG}$ methylation that is observed in these tumor samples.

A recent study that explored the correlation of methylation, transcription and chromatin structure found that in normal tissues, methylation $1 \mathrm{~kb}$ upstream of the TSS was most correlated with gene silencing, but in cancer, there was a shift to the TSS [18]. Given that the target regions described here were primarily in the promoter region and overlapping or close to the TSS, it is reasonable to hypothesize that these genes are likely being silenced in cancer.

Despite the diversity of normal tissue types in the WGBS discovery cohort, the MSRE-PCR target regions identified here were nearly universally unmethylated, with an average methylation within these $\mathrm{CpG}$ dense region of only $1.5 \%$. The very low levels of methylation in these regions in normal tissues suggest that there are stringent and vigilant mechanisms in place to keep these regions clear of methylation marks. The hypermethylation of these target regions in cancer thus represents a departure from a precisely maintained unmethylated state and indicates a change that is generally conserved to some extent across the diverse tumor types used in this study. This evidence suggests that the genes associated with these target regions

\section{A}

Volcano Plot: MSRE-PCR Amplicons in Diverse Tumors vs. Normal Tissues

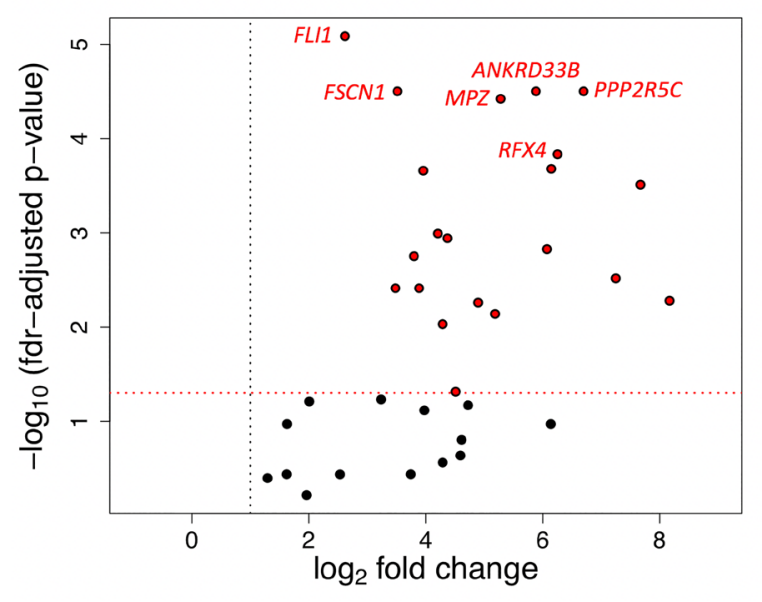

were acquiring epigenetic marks in cancer that are likely silencing these genes - changes that may well be relevant to oncogenic transformation and/or disease progression.

The set of genes closest to or overlapping the target regions showed a significant enrichment in neuronal development and synapse function which underscore a neuronal theme to this set of target genes. Interestingly, it has been reported that the neurofilament genes $N E F H$, $N E F L$ and NEFM, gene products that comprise the major cytoskeletal component of neurons, are frequently silenced via promoter methylation in a variety of cancers including breast, pancreas, gastric and colon and this gene inactivation is correlated with disease progression and adverse clinical parameters [19]. Reintroducing NEFH expression in breast cancer cells reduced invasiveness, suggesting that expression of neuronal filaments in cancer induced a rearrangement of the cytoskeleton that reduced motility and migration. Another study showed that inhibition of epigenetic modification enzymes, including DNA methyl transferases (DNMTs), induced a postmitotic, neuronal-like terminal differentiation in different types of cancer cells [20]. These studies, taken together with the targeted promoter methylation of neuronalrelated genes shown here, underscore an emerging pattern of tumor-associated silencing of neuronal genes, a mechanism which may facilitate the maintenance of an undifferentiated, proliferative state with the oncogenic characteristics of mobility and invasiveness.

The target regions identified here are not only differentially methylated in tumors versus normal tissues,

B Amplicons Significantly Elevated in
Diverse Tumors vs. Normal Tissue

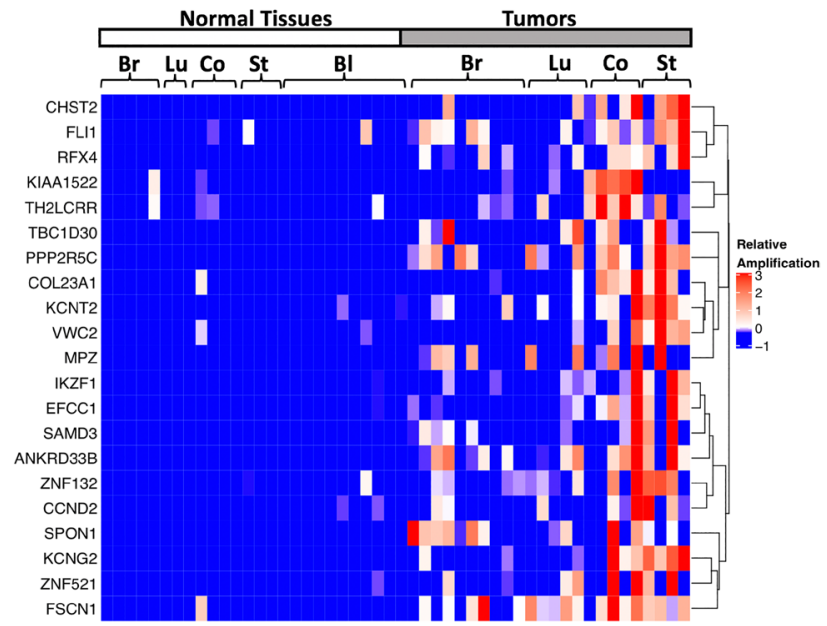

Figure 8: Multiplexed MSRE-PCR of the target regions in genomic DNAs from diverse tumors versus normal tissue. (A) Volcano plot of $-\log _{10}$ FDR-corrected $p$-values (Mann-Whitney $U$ test) versus $\log 2$ fold-change in the training set. All 35 amplicons have a linear fold-change $>2$ fold elevated in the tumor DNA samples (indicated with the vertical dotted black line). The 21 amplicons with FDR corrected $p$-value $<0.05$ (horizontal dotted red line) are indicated with red points. The 6 amplicons with the lowest $p$-values in the training set are labeled with the gene symbol for the overlapping gene or promoter region. (B) Hierarchical clustering of the 21 amplicons with FDR corrected $p$-value $<0.05$ in the training set with the color indicating the relative level of amplification. The heatmap is supervised in the x-dimension, with samples arranged by tissue type (Br: breast, LU: lung, Co: colon, St: stomach, Bl: blood) and unsupervised in the $\mathrm{y}$-dimension, with amplicons labeled by the gene symbol of the overlapping gene or promoter region. 
but also have the unique characteristic of very low average methylation levels $<2.5 \%$ in normal tissues. In the context of MSRE-PCR, this hypomethylation status in normal DNA in theory allows for the nearly complete digestion of normal DNA, as only methylated MSRE sites will be protected from digestion. Furthermore, in addition to an extremely low levels of methylation in normal tissues, each amplicon contains multiple $\mathrm{CpGs}$ that are interrogated by one of the MSREs. For successful amplification, all of the
MSRE sites on a single molecule need to be methylated and protected from digestion for amplification of that molecule to proceed. Thus, the hypomethylation in normal tissues taken together with multiple MSRE sites within these regions should facilitate the digestion of the normal DNA relative to tumor-derived DNA and consequently enhance the specificity of the assay. Indeed, here we observed that all 35 amplicons tested in the multiplexed MSRE-PCR assay exhibited $>2$-fold elevated amplification in the

\section{7-marker model: Training and Testing Sets}

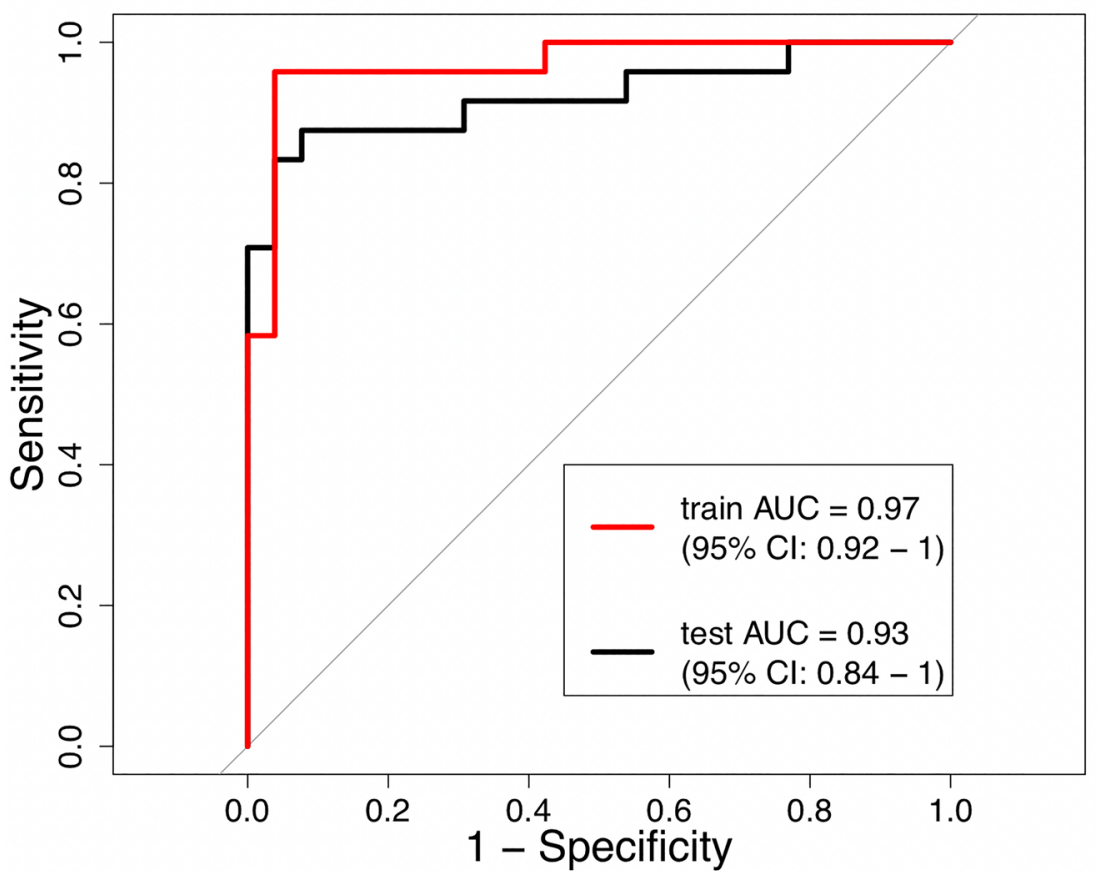

Figure 9: Receiver operating characteristic (ROC) curves of the 7-marker logistic regression model in distinguishing between tumor and normal tissue DNA. The performance of the model in the training set $(n=50)$ is shown in red (AUC $=0.97,95 \%$ CI: $0.92-1)$ and the independent test set is shown in black (AUC $=0.93,95 \% \mathrm{CI}: 0.84-1)$.
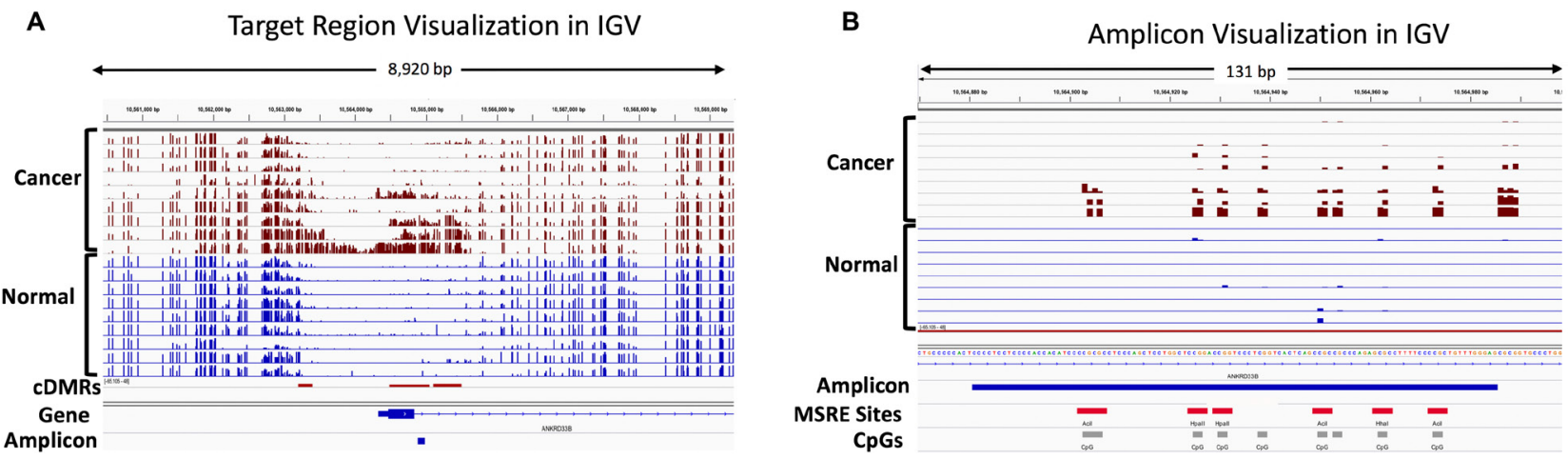

Figure 10: IGV visualization of the WGBS discovery cohort showing hypermethylated cDMRs and the location of an amplicon in the $\boldsymbol{A N K R D 3 3 B}$ gene. Percent methylation for individual CpGs is indicated with bar graphs for each of the cancer samples (maroon) and normal tissue samples (blue) used in the WGBS data analysis. (A) The location of 3 hypermethylated cDMRs in the promoter and gene body of $A N K R D 33 B$ is indicated with the red rectangles in the cDMR track and the location of the MSRE-PCR amplicon is shown in blue in the Amplicon track. (B) A zoomed-in view of the MSRE-PCR amplicon shows the position of the individual CpGs (grey) and MSRE sites (red) within this 106 bp amplicon. 
tumor samples, with the majority of the amplicons (27 of 35 ) exhibiting $>10$-fold elevation.

The multiplexed MSRE-PCR feasibility study on diverse tumor and normal tissue genomic DNAs reported here identified 21 genes with significantly elevated amplification in this assay and further defined a 7-marker logistic regression model that distinguished between tumor and normal tissue with an AUC of 0.94 in the independent test set. The 7 markers used in this model correspond to amplicons contained within the gene or promoter region of ANKRD33B, CHST2, SPON1, PPP2R5C, KCNG2, KIAA1522 and TH2LCRR. Consistent with the neuronal theme identified in the gene set enrichment analysis, SPON1 is a cell-surface adhesion molecule involved in sensory neuron attachment; its expression has been associated with metastatic progression in osteosarcomas [21]. Both PPP2R5C and KIAA1522 have also been associated with cancer progression and/or prognosis. $P P P 2 R 5 C$ encodes an isoform of the $\mathrm{B}$ regulatory subunit of protein phosphatase $2 \mathrm{~A}$, a serine/threonine phosphatase widely implicated in the regulation of mitogenic pathways [22]. Aberrant expression of KIAA1522 has been observed in non-small cell lung cancer and is associated with poor outcome [23]. ANKRD33B, encoding ankyrin repeat domain $33 \mathrm{~B}$, has been shown to be differentially methylated in fetal lung in association with nicotine exposure in utero [24].

The multiplexed MSRE-PCR assay described here interrogated 35 target regions using endpoint PCR and quantitated amplification via sequencing. While this strategy is convenient to assess many amplicons simultaneously, the relatively small number of features required for sample classification enables the development of a qPCR-based assay that interrogates fewer target regions in individual qPCR reactions or as a multiplexed reaction with fluorescent reporter probes. In the multiplexed MSRE-PCR assay described here, there is some variability in the performance of the individual amplicons, with some showing only modest levels of elevated amplification in tumor DNA samples. The relatively poor performance of some amplicons may be attributable to the challenges of moving from the WGBS platform to a PCR platform along with variable amplification efficiencies in these GC-rich regions, particularly in the context of a highly multiplexed amplification reaction. While the multiplexed endpoint MSRE-PCR assay showed that 27 of the 35 target regions had $>10$-fold elevated amplification in the tumor DNA samples, this fold-change may well be enhanced by utilizing a qPCR strategy that quantitates amplification in real time. The robust elevated amplification of tumor DNA observed here in genomic DNA samples lays the foundation for applying multiplexed MSRE-PCR strategies for the detection of tumor DNA in more complex sample types such as cfDNA in blood, where the fraction of tumor-derived DNA may be limiting.
While this study included multiple normal tissue and tumor types, it is nevertheless limited in the scope of tumor types examined, with the cohort for the MSREPCR assay encompassing breast, stomach, lung, and colon tumors. While this cohort is diverse, it is by no means comprehensive and thus the utility of the MSRE-PCR assay defined here and the conservation of the cDMRs in a large and more comprehensive cohort remains to be investigated.

The data presented here define genomic target regions for MSRE-PCR detection of tumor DNA and establish the utility of these target regions in a multiplexed MSRE-PCR assay. MSRE-PCR enables the selective amplification of hypermethylated tumor-derived DNA while avoiding harsh bisulfite treatment and thus is a strategy ideally suited for the challenges of non-invasive detection. The hypermethylated MSRE-PCR target regions defined here may be useful in the development of noninvasive tests for tumor detection and/or monitoring.

\section{MATERIALS AND METHODS}

\section{WGBS data analysis}

WGBS raw data was obtained from the NCBI Sequence Read Archive (SRA) database [25]. 48 individual SRA runs representing 18 biological samples were analyzed (Table 1 and Supplementary Table 1). Multiple runs for the same biological sample were combined to optimize coverage. The SRA Toolkit [25] was used to extract fasta files and fastQC [26] was used to asses the quality of the raw data. Poor quality sequences and adapters were trimmed with Trimmomatic [27] and the quality of the trimmed reads was assessed with fastQC. The bisulfite converted reads were mapped to hg38 and CpG methylation calls were extracted using Bismark [28]. Differentially methylated Regions (DMRs) were identified with Metilene [14], filtering the output for minimum mean methylation difference of 0.15 , minimum size of 80 nucleotides and $Q$-value $<0.01$. Summary figures and annotation of DMRs was performed in R using the package methylKit [29]. Target regions were defined as hypermethylated DMRs with a mean methylation $<2.5 \%$ in normal samples.

\section{MSRE-PCR}

PCR primers were designed using Benchling [30] with the considerations that the amplicons have at least 2 MSRE sites, an amplicon size $<150 \mathrm{bp}$ and PCR primer melting temperatures of approximately 61 degrees (Supplementary Table 3). Descriptive characteristics of the 47 target regions and 3 controls in the multiplexed MSREPCR panel such as the genomic coordinates and the number of $\mathrm{CpGs}$ and MSRE sites within each amplicon is detailed in Supplementary Table 2. Genomic DNA 
samples from lung, breast, stomach and colon tumors and normal tissues were obtained from Biochain (Newark, CA, catalog numbers D8235152-1, D8235086-1, D82350901, D8234148-1, D8235248-1) (Supplementary Table 5). Oligonucleotides were synthesized at IDT (Coralville, Iowa). DNA samples (20 ng) were digested with 5 Units each of 5 MSREs: HhaI, HpaII, HpyCH4IV, AciI and BstUI, all from New England Biolabs (Ipswich, MA).

The amplification of the multiplexed panel was done in several steps, incorporating a hybrid selection step to enrich for target sequences and minimize off-target amplification. Briefly, $5 \mathrm{ng}$ of digested genomic DNA was subjected to 10 cycles $\left(94^{\circ} \mathrm{C} 3 \mathrm{~min}\left[95^{\circ} \mathrm{C} 30 \mathrm{~s}, 61^{\circ} \mathrm{C}\right.\right.$ $\left.30 \mathrm{~s}, 72^{\circ} \mathrm{C} 30 \mathrm{~s}\right] \times 10$ ) of multiplexed PCR amplification using the 50 MSRE-PCR primer pairs and Platinum II Hot-Start Green PCR Master Mix (ThermoFisher Scientific). The preamplification reaction was hybridized to a pool of 50 biotinylated oligonucleotides (custom IDT xGen Lockdown probe pool, Supplementary Table 4) complimentary to internal sequences within each amplicon. The xGen Hybridization and wash kit (IDT, Coralville, Iowa) was used for target enrichment according to the manufacturer's protocol. After hybrid selection, the magnetic beads containing the captured target amplicons were resuspended in $10 \mathrm{uL}$ of $\mathrm{dH}_{2} \mathrm{O}$ and $5 \mathrm{uL}$ was used in a $20 \mathrm{uL}$ amplification reaction with the 50 multiplexed MSRE primer pairs for 35 cycles $\left(94^{\circ} \mathrm{C} 3 \mathrm{~min}\left[95^{\circ} \mathrm{C} 30 \mathrm{~s}\right.\right.$, $\left.\left.61^{\circ} \mathrm{C} 30 \mathrm{~s}, 72^{\circ} \mathrm{C} 30 \mathrm{~s}\right] \times 35\right)$.

Amplification products were sequenced on the Illumina platform at Genewiz (South Plainfield, NJ, USA) at a depth of 50,000 reads per sample. FastQC was used to assess the quality of the raw data. Adapters and poor quality sequences were trimmed with Trimmomatic. Reads were mapped using Bowtie2 [31] and quantitation of reads mapping to the target regions was carried out using Samtools [32]. Samples were normalized to an internal control amplicon (Control3) corresponding to a genomic region that was not differentially methylated in the WGBS discovery cohort (Supplementary Table 2). Digestion by the MSREs was assessed using a different control amplicon (Control1) that had MSRE sites and was consistently unmethylated across sample types in the WGBS discovery cohort and thus would be predicted to be completely digested. Statistical analysis was carried out in R [33] and heatmaps were generated using the $\mathrm{R}$ package ComplexHeatmap [34]. Summary statistics from the multiplexed MSRE-PCR assay are available in Supplementary Table 6. Summary statistics for the 1,294 target regions are in Supplementary Table 7.

\section{Predictive modeling and validation}

The MSRE-PCR data was randomly partitioned into training $(n=50)$ and testing $(n=50)$ sets and the $\mathrm{R}$ package glmnet [16] was used to fit a logistic regression model on the training set, employing a lasso penalty controlled by the tuning parameter lambda. The optimal value of lambda was determined by K-fold cross-validation with the parameters family set to "binomial", the number of folds set to 3 and ROC AUC as the type of measurement for model evaluation. The largest value of lambda which yielded an AUC within one standard error of the maximum AUC was chosen, yielding a logistic regression model with 7 markers (Supplementary Table 5). The 7-marker model was validated on the independent test set and ROC AUC and confidence intervals were determined using the $\mathrm{pROC} R$ package [35].

\section{Data accessibility}

The WGBS data used in this study was accessed from the NCBI SRA database. Individual accessions used in this study are detailed in Supplementary Table 1. Oligonucleotide sequences used for PCR and hybrid selection are available in Supplementary Tables 2 and 3. MSRE-PCR data is available upon request.

\section{Abbreviations}

MSRE: Methylation Sensitive Restriction Enzyme; MSRE-PCR: Methylation Sensitive Restriction Enzyme PCR; DMR: Differentially Methylated Region; cDMR: Differentially Methylated Region in cancer; WGBS: Whole Genome Bisulfite Sequencing; cfDNA: Circulating cell-free DNA; ctDNA: Circulating tumor DNA; SRA: Sequence Read Archive; PCA: Principal Component Analysis; ROC: Receiver Operating Characteristic; AUC: Area Under the Curve.

\section{Author contributions}

The bioinformatics analysis was carried out by $\mathrm{KC}$ under the supervision of BH. The MSRE-PCR was carried out by $\mathrm{KC}$. Both authors participated in the preparation and revision of the manuscript. All authors read and approved the final manuscript.

\section{CONFLICTS OF INTEREST}

Authors have no conflicts of interest to declare.

\section{FUNDING}

This study was primarily carried out by $\mathrm{KC}$ on publicly available data in the context of an advanced degree at Johns Hopkins University, Advanced Academic Programs without additional external funding.

\section{REFERENCES}

1. Klutstein M, Nejman D, Greenfield R, Cedar H. DNA Methylation in Cancer and Aging. Cancer Res. 2016; 
76:3446-50. https://doi.org/10.1158/0008-5472.CAN-153278. [PubMed]

2. Esteller M, Herman JG. Cancer as an epigenetic disease: DNA methylation and chromatin alterations in human tumours. J Pathol. 2002; 196:1-7. https://doi.org/10.1002/ path.1024. [PubMed]

3. Baylin SB. DNA methylation and gene silencing in cancer. Nat Clin Pract Oncol. 2005; 2:S4-11. https://doi. org/10.1038/ncponc0354. [PubMed]

4. Pan Y, Liu G, Zhou F, Su B, Li Y. DNA methylation profiles in cancer diagnosis and therapeutics. Clin Exp Med. 2018; 18:1-14. https://doi.org/10.1007/s10238-017-0467-0. [PubMed]

5. Bao X, Anastasov N, Wang Y, Rosemann M. A novel epigenetic signature for overall survival prediction in patients with breast cancer. J Transl Med. 2019; 17:380. https://doi.org/10.1186/s12967-019-2126-6. [PubMed]

6. Barault L, Amatu A, Siravegna G, Ponzetti A, Moran S, Cassingena A, Mussolin B, Falcomata C, Binder AM, Cristiano C, Oddo D, Guarrera S, Cancelliere C, et al. Discovery of methylated circulating DNA biomarkers for comprehensive non-invasive monitoring of treatment response in metastatic colorectal cancer. Gut. 2018; 67:1995-2005. https://doi.org/10.1136/gutjnl-2016-313372. [PubMed]

7. Shirahata A, Sakuraba K, Goto T, Saito M, Ishibashi K, Kigawa G, Nemoto H, Hibi K. Detection of vimentin (VIM) methylation in the serum of colorectal cancer patients. Anticancer Res. 2010; 30:5015-8. [PubMed]

8. Darst RP, Pardo CE, Ai L, Brown KD, Kladde MP. Bisulfite sequencing of DNA. Curr Protoc Mol Biol. 2010; Chapter 7:1-17. https://doi.org/10.1002/0471142727.mb0709s91. [PubMed]

9. Hernandez HG, Tse MY, Pang SC, Arboleda H, Forero DA. Optimizing methodologies for PCR-based DNA methylation analysis. Biotechniques. 2013; 55:181-97. https://doi.org/10.2144/000114087. [PubMed]

10. Melnikov AA, Gartenhaus RB, Levenson AS, Motchoulskaia NA, Levenson Chernokhvostov VV. MSREPCR for analysis of gene-specific DNA methylation. Nucleic Acids Res. 2005; 33:e93. https://doi.org/10.1093/ nar/gni092. [PubMed]

11. Beikircher G, Pulverer W, Hofner M, Noehammer C, Weinhaeusel A. Multiplexed and Sensitive DNA Methylation Testing Using Methylation-Sensitive Restriction Enzymes "MSRE-qPCR". Methods Mol Biol. 2018; 1708:407-24. https://doi.org/10.1007/978-1-49397481-8_21. [PubMed]

12. Suehiro Y, Hashimoto S, Higaki S, Fujii I, Suzuki C, Hoshida T, Matsumoto T, Yamaoka Y, Takami T, Sakaida I, Yamasaki T. Blood free-circulating DNA testing by highly sensitive methylation assay to diagnose colorectal neoplasias. Oncotarget. 2018; 9:16974-87. https://doi. org/10.18632/oncotarget.24768. [PubMed]
13. Wielscher M, Vierlinger K, Kegler U, Ziesche R, Gsur A, Weinhausel A. Diagnostic Performance of Plasma DNA Methylation Profiles in Lung Cancer, Pulmonary Fibrosis and COPD. EBioMedicine. 2015; 2:929-36. https://doi. org/10.1016/i.ebiom.2015.06.025. [PubMed]

14. Juhling F, Kretzmer H, Bernhart SH, Otto C, Stadler PF, Hoffmann S. metilene: fast and sensitive calling of differentially methylated regions from bisulfite sequencing data. Genome Res. 2016; 26:256-62. https://doi. org/10.1101/gr.196394.115. [PubMed]

15. Supek F, Bosnjak M, Skunca N, Smuc T. REVIGO summarizes and visualizes long lists of gene ontology terms. PLoS One. 2011; 6:e21800. https://doi.org/10.1371/ journal.pone.0021800. [PubMed]

16. Friedman J, Hastie T, Tibshirani R. Regularization Paths for Generalized Linear Models via Coordinate Descent. J Stat Softw. 2010; 33:1-22. [PubMed]

17. Vidal E, Sayols S, Moran S, Guillaumet-Adkins A, Schroeder MP, Royo R, Orozco M, Gut M, Gut I, Lopez-Bigas N, Heyn $\mathrm{H}$, Esteller M. A DNA methylation map of human cancer at single base-pair resolution. Oncogene. 2017; 36:5648-57. https://doi.org/10.1038/onc.2017.176. [PubMed]

18. Ando M, Saito Y, Xu G, Bui NQ, Medetgul-Ernar K, Pu M, Fisch K, Ren S, Sakai A, Fukusumi T, Liu C, Haft S, Pang $\mathrm{J}$, et al. Chromatin dysregulation and DNA methylation at transcription start sites associated with transcriptional repression in cancers. Nat Commun. 2019; 10:2188. https:// doi.org/10.1038/s41467-019-09937-w. [PubMed]

19. Calmon MF, Jeschke J, Zhang W, Dhir M, Siebenkas C, Herrera A, Tsai HC, O’Hagan HM, Pappou EP, Hooker CM, $\mathrm{Fu}$ T, Schuebel KE, Gabrielson E, et al. Epigenetic silencing of neurofilament genes promotes an aggressive phenotype in breast cancer. Epigenetics. 2015; 10:622-32. https://doi. org/10.1080/15592294.2015.1050173. [PubMed]

20. Lei A, Chen L, Zhang M, Yang X, Xu L, Cao N, Zhang Z, Cao Y. EZH2 Regulates Protein Stability via Recruiting USP7 to Mediate Neuronal Gene Expression in Cancer Cells. Front Genet. 2019; 10:422. https://doi.org/10.3389/ fgene.2019.00422. [PubMed]

21. Chang H, Dong T, Ma X, Zhang T, Chen Z, Yang Z, Zhang Y. Spondin 1 promotes metastatic progression through Fak and Src dependent pathway in human osteosarcoma. Biochem Biophys Res Commun. 2015; 464:45-50. https:// doi.org/10.1016/j.bbrc.2015.05.092. [PubMed]

22. Kauko O, Westermarck J. Non-genomic mechanisms of protein phosphatase $2 \mathrm{~A}(\mathrm{PP} 2 \mathrm{~A})$ regulation in cancer. Int J Biochem Cell Biol. 2018; 96:157-64. https://doi. org/10.1016/j.biocel.2018.01.005. [PubMed]

23. Liu YZ, Yang H, Cao J, Jiang YY, Hao JJ, Xu X, Cai Y, Wang MR. KIAA1522 is a novel prognostic biomarker in patients with non-small cell lung cancer. Sci Rep. 2016; 6:24786. https://doi.org/10.1038/srep24786. [PubMed]

24. Chhabra D, Sharma S, Kho AT, Gaedigk R, Vyhlidal CA, Leeder JS, Morrow J, Carey VJ, Weiss ST, Tantisira 
$\mathrm{KG}$, DeMeo DL. Fetal lung and placental methylation is associated with in utero nicotine exposure. Epigenetics. 2014; 9:1473-84. https://doi.org/10.4161/15592294.2014.971593. [PubMed]

25. Leinonen R, Sugawara H, Shumway M, International Nucleotide Sequence Database C. The sequence read archive. Nucleic Acids Res. 2011; 39:D19-21. https://doi. org/10.1093/nar/gkq1019. [PubMed]

26. Andrews S. FastQC: a quality control tool for high throughput sequence data. 2010.

27. Bolger AM, Lohse M, Usadel B. Trimmomatic: a flexible trimmer for Illumina sequence data. Bioinformatics. 2014; 30:2114-20. https://doi.org/10.1093/bioinformatics/btu170. [PubMed]

28. Krueger F, Andrews SR. Bismark: a flexible aligner and methylation caller for Bisulfite-Seq applications. Bioinformatics. 2011; 27:1571-2. https://doi.org/10.1093/ bioinformatics/btr167. [PubMed]

29. Akalin A, Kormaksson M, Li S, Garrett-Bakelman FE, Figueroa ME, Melnick A, Mason CE. methylKit: a comprehensive $\mathrm{R}$ package for the analysis of genome-wide DNA methylation profiles. Genome Biol. 2012; 13:R87. https://doi.org/10.1186/gb-2012-13-10-r87. [PubMed]

30. Benchling. Benchling [Biology Software].

31. Langmead B, Salzberg SL. Fast gapped-read alignment with Bowtie 2. Nat Methods. 2012; 9:357-9. https://doi. org/10.1038/nmeth.1923. [PubMed]
32. Li H, Handsaker B, Wysoker A, Fennell T, Ruan J, Homer N, Marth G, Abecasis G, Durbin R; 1000 Genome Project Data Processing Subgroup. The Sequence Alignment/Map format and SAMtools. Bioinformatics. 2009; 25:2078-9. https://doi.org/10.1093/bioinformatics/btp352. [PubMed]

33. Team RC. R: A language and environment for statistical computing. (Vienna, Austria: R Foundation for Statistical Computing). 2018.

34. Gu Z, Eils R, Schlesner M. Complex heatmaps reveal patterns and correlations in multidimensional genomic data. Bioinformatics. 2016; 32:2847-9. https://doi.org/10.1093/ bioinformatics/btw313. [PubMed]

35. Robin X, Turck N, Hainard A, Tiberti N, Lisacek F, Sanchez JC, Muller M. pROC: an open-source package for R and S+ to analyze and compare ROC curves. BMC Bioinformatics. 2011; 12:77. https://doi.org/10.1186/1471-2105-12-77. [PubMed]

36. Jenkinson G, Pujadas E, Goutsias J, Feinberg AP. Potential energy landscapes identify the information-theoretic nature of the epigenome. Nat Genet. 2017; 49:719-29. https://doi. org/10.1038/ng.3811. [PubMed]

37. Heyn H, Vidal E, Ferreira HJ, Vizoso M, Sayols S, Gomez A, Moran S, Boque-Sastre R, Guil S, Martinez-Cardus A, Lin CY, Royo R, Sanchez-Mut JV, et al. Epigenomic analysis detects aberrant super-enhancer DNA methylation in human cancer. Genome Biol. 2016; 17:11. https://doi. org/10.1186/s13059-016-0879-2. [PubMed] 